EGU2020-18944

https://doi.org/10.5194/egusphere-egu2020-18944

EGU General Assembly 2020

(c) Author(s) 2020. This work is distributed under

the Creative Commons Attribution 4.0 License.

\title{
Tools for fast analysis of InSAR based displacement maps
}

Oriol Monserrat ${ }^{1}$, Anna Barra ${ }^{1}$, Roberto Tomás ${ }^{2}$, José Navarro ${ }^{1}$, Lorenzo Solari ${ }^{1}$, Gerardo Herrera ${ }^{3}$, and Michele Crosetto ${ }^{1}$

${ }^{1}$ CTTC, Remote Sensing, Castelldefels, Spain (anna.barra@cttc.es)

${ }^{2}$ Departamento de Ingeniería Civil, Universidad de Alicante, Alicante, Spain

${ }^{3}$ Geohazards InSAR Laboratory and Modeling Group, Instituto Geológico y Minero de España (IGME), Madrid, Spain

The use of satellite interferometry (InSAR) is exponentially growing for the detection and monitoring of geohazard related movements. InSAR technique allows to process large areas and to extract high number of displacement measurements at low cost. By the way, the outputs consist of high volumes of information whose interpretation can be complex and time-consuming, mostly for users who are not familiar with radar data. Moreover, the use of InSAR have been moving from local to national, and now we are going towards a European application. In this scenario, the development of methodologies and tools to automatize the extraction of significant information and to facilitate the interpretation of the results, is more and more needed in order to increase their operational use. In this work we present a series of tools developed in the framework of the projects DEMOS (CGL2017- 83704-P), Momit (S2R-H2020/777630), Safety (ECHO/SUB/2015/718679) and U-Geohaz (UCPM-2017-PP-AG/783169). The so-called ADA (Active Displacement Areas) tools have been developed with the aim of ease the management, the use and the interpretation of wide areas results. Starting from the semi-automatic extraction of the most significant Active Displacement Areas (ADAFinder tool) we move to an automatic preliminary assessment of the phenomena that is behind the detected movement (ADAClassifier tool). All these tools go in the same direction of the European Ground Motion Service (EU-GMS) project, which will provide consistent, regular and reliable information regarding natural and anthropogenic ground motion phenomena all over Europe. 